\title{
RESULTS OF THE 2008 INTERNATIONAL BUTTERFLY COUNTINSASKATCHEWAN
}

\author{
MIKE GOLLOP, 51 Welker Crescent, Saskatoon, SK, S7H 3M3 and ANNA
} LEIGHTON, 328 Saskatchewan Crescent West, Saskatoon, SK, S7M OA4

Nine International Butterfly Counts (1 JC) were conducted in Saskatchewan in 2008, the same as in 2007 and one more than in 2006. Counts in 2008 were conducted at Bjorkdale, Fort Qu'Appelle, Last Mountain Lake National Wildlife Area, Nisbet Forest, Pasquia Hills, Preeceville, Regina, Saskatoon and Waskesiu River. The Waskesiu River count was last conducted in 2006. Duck Mountain Park was the only count done in 2007 that was not repeated in 2008. Count statistics are presented in Table 1 , and count results in Table 2.

There were 66 species recorded in 2008 compared to 60 in 2007, 62 in 2006 and 57 in 2005. ${ }^{1,2,3}$ The total number of butterflies counted was 4983 compared to 4007 in 2007, 3669 in 2006, and 6972 in 2005, of which 5389 were Painted Ladies. Butterflies per party-hour (ph) is used as a measure to relate butterfly numbers to observer effort so as to provide a comparable index of abundance over years. Butterflies per party-hour was 62 in 2008 for the nine counts compared to 48 in 2007 and 2006 and 86 in 2005, although again, if Painted Ladies are removed from the 2005 count, the butterflies per party-hour would be only 27 . It appears from this that overall numbers of butterflies were up in 2008 but such provincial averaging may mask local results. In general the more southerly counts and those held earlier in the year had significantly fewer butterflies than did the later counts along the forest fringe.
Count conditions in 2008 were variable. Temperatures ranged from a low of $16^{\circ}$ at Waskesiu River to a high of $28^{\circ}$ at Pasquia Hills. Wind speeds exceeding $30 \mathrm{~km} / \mathrm{hr}$ at Pasquia Hills, Preeceville, Regina and Saskatoon may have had some negative influence on the number of open field butterflies that would have been seen. Lack of sun (< $50 \%$ of count period) may also have negatively influenced counts at Last Mountain Lake and Preeceville.

Species totals on the counts ranged from 15 at Last Mountain Lake to 31 at Bjorkdale and averaged 22 for the nine counts. This was down from 24 in 2007 but up from 20 in 2006 and 18 in 2005. Anything more than 20 species is very respectable for a single-day butterfly count and over 30 is exceptional. Only five counts exceeded 20 species in 2008, whereas seven did in 2007.

Numbers of individuals ranged from 139 at Last Mountain Lake to 1733 at Pasquia Hills. This compares to a range of 153 at Last Mountain Lake and 1013 at Bjorkdale in 2007 and 115 at Fort Qu'Appelle and 1152 at Eastend in 2006. The number of individuals per party-hour ranged from 15 at Last Mountain lake and Saskatoon to 173 at Pasquia Hills. This compares with a range from 22 at Last Mountain Lake to 127 at Bjorkdale in 2007 and from 19 at Fort Qu'Appelle to 164 at Eastend in 2006. 


\begin{tabular}{|c|c|c|c|c|c|c|c|c|c|c|}
\hline 을 $\frac{\bar{c}}{\bar{c}}$ & $\infty$ & $\mid$ & $\infty$ & $\mid \begin{array}{c}\hat{n} \\
1 \\
\hat{n} \\
n\end{array}$ & $\mid \begin{array}{l}m \\
\frac{m}{1} \\
0 \\
\frac{1}{6}\end{array}$ & 畺 & 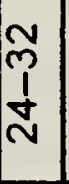 & $\begin{array}{l}\tilde{m} \\
0 \\
0 \\
0\end{array}$ & $\mid \begin{array}{c}0 \\
\frac{1}{1} \\
\infty\end{array}$ & pe \\
\hline $\begin{array}{ll}\dot{Q} & 0 \\
\stackrel{E}{E} & \dot{D} \\
\stackrel{D}{D} & 0\end{array}$ & $\frac{0}{1}$ & $\mid$ & $\stackrel{m}{w}$ & $\left|\begin{array}{c}\mathscr{O} \\
\stackrel{1}{N} \\
\stackrel{N}{N}\end{array}\right|$ & $\left|\begin{array}{c}o \\
N \\
r \\
r\end{array}\right|$ & 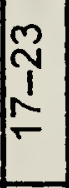 & 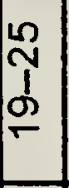 & 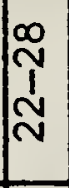 & $\mid \begin{array}{l}2 \\
N \\
1 \\
\infty \\
N\end{array}$ & 象 \\
\hline $\begin{array}{cc}5 & \varepsilon \\
\text { s } & 0 \\
\alpha^{\circ} & \end{array}$ & $\stackrel{\sim}{N}$ & (ొ) & R & $\stackrel{w}{\sim}$ & $\left|\begin{array}{l}0 \\
0 \\
1 \\
0 \\
\end{array}\right|$ & $n$ & $\stackrel{\stackrel{\sim}{N}}{\mathbb{v}}$ & $\infty$ & $\frac{10}{1}$ & 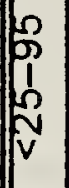 \\
\hline $\begin{array}{cc}{ }^{*} & \\
\sim & \frac{E}{\sigma} \\
0 & \\
0 & \end{array}$ & $\frac{8}{0}$ & $\mathscr{\infty}$ & 음 & $\mid \begin{array}{l}n \\
\frac{1}{1} \\
\frac{1}{10}\end{array}$ & 战 & 8 & 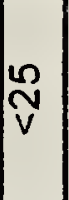 & 음 & $\begin{array}{l}n \\
\frac{1}{1} \\
\frac{1}{n} \\
10\end{array}$ & 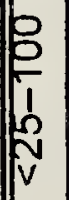 \\
\hline 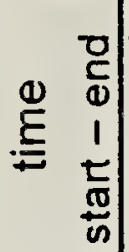 & $\mid \begin{array}{l}0 \\
\frac{0}{1} \\
\frac{1}{1} \\
0 \\
0 \\
0\end{array}$ & $\mid \begin{array}{l}0 \\
0 \\
10 \\
1 \\
0 \\
0 \\
0 \\
0\end{array}$ & $\mid \begin{array}{l}8 \\
0 \\
0 \\
1 \\
0 \\
0 \\
0 \\
0\end{array}$ & $\mid$\begin{tabular}{l}
8 \\
0 \\
\hdashline \\
1 \\
0 \\
0 \\
0 \\
0
\end{tabular} & 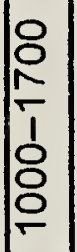 & $\mid$\begin{tabular}{l}
8 \\
0 \\
\hdashline \\
1 \\
8 \\
0 \\
0 \\
0
\end{tabular} & 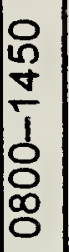 & 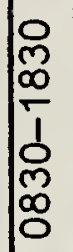 & $\begin{array}{l}8 \\
0 \\
1 \\
1 \\
8 \\
8 \\
0\end{array}$ & 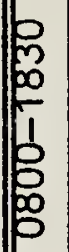 \\
\hline $\begin{array}{ll}\vec{a} & \\
\frac{E}{x} & \bar{J} \\
\end{array}$ & 0 & 0 & m & 18 & 0 & m & & $\stackrel{\sim}{\sim}$ & 0 & \\
\hline $\begin{array}{ll}c & \\
& \\
\frac{E}{x} & 0 \\
\end{array}$ & $\infty$ & $\begin{array}{l}\infty \\
\dot{v} \\
\end{array}$ & $\infty$ & $\stackrel{m}{\stackrel{w}{F}}$ & $\underset{0}{+}$ & 10 & n & $m$ & $\infty$ & \\
\hline 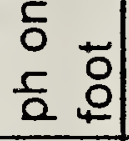 & 0 & 음 & & $\mathscr{\sim}$ & $\left|\begin{array}{l}n \\
\infty \\
0\end{array}\right|$ & $\sim$ & $\mid$ & $N$ & $\omega$ & \\
\hline 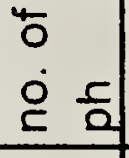 & or & 웅 & $r$ & $\approx$ & $\left|\begin{array}{l}n \\
\infty \\
0\end{array}\right|$ & $\sigma$ & $\mid \begin{array}{l}n \\
0\end{array}$ & 으 & $\omega$ & 仍 \\
\hline $\begin{array}{ll} & \\
0 & 0 \\
\subseteq & 0 \\
\end{array}$ & $m$ & N & 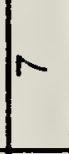 & $F$ & o & $r$ & $m$ & F & m & pr \\
\hline 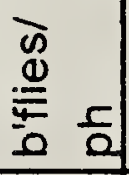 & $\neq$ & I & $\hat{N}$ & $\cong$ & In & mे & 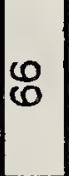 & a & p & Fo \\
\hline 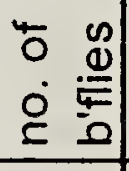 & $\stackrel{n}{2}$ & $\overline{\mathscr{m}}$ & $\stackrel{\infty}{\infty}$ & $\mid \begin{array}{l}\infty \\
\tilde{m}\end{array}$ & m & 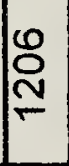 & \begin{tabular}{l}
$\infty$ \\
$\sim$ \\
\multirow{y}{*}{}
\end{tabular} & $\stackrel{m}{\stackrel{m}{R}}$ & $\frac{\infty}{8}$ & 占 \\
\hline 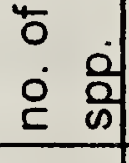 & $\approx$ & $\mathscr{R}$ & $\stackrel{\sim}{\sim}$ & $\stackrel{\sim}{\sim}$ & 10 & $\bar{m}$ & $\stackrel{\infty}{-}$ & প্লি & 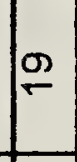 & 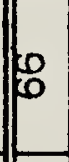 \\
\hline 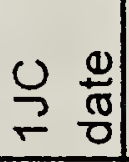 & $\begin{array}{l}z \\
\text { z } \\
\text { s }\end{array}$ & $\begin{array}{l}z \\
\\
\sigma \\
\end{array}$ & $\frac{z}{i}$ & 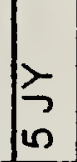 & $\begin{array}{l}2 \\
\infty \\
\infty\end{array}$ & $\begin{array}{l}2 \\
\infty \\
\infty\end{array}$ & 文 & $\underset{N}{N}$ & $\mid \begin{array}{l}2 \\
\alpha \\
6\end{array}$ & fos \\
\hline 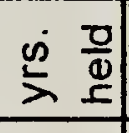 & $\mp$ & $\stackrel{m}{\square}$ & $\stackrel{\infty}{\sim}$ & \pm & $N$ & $\infty$ & 12 & $\infty$ & 19 & \\
\hline \begin{tabular}{ll}
$\mathcal{U}$ & $\stackrel{0}{E}$ \\
\hdashline & $\frac{0}{c}$
\end{tabular} & ${ }_{3}^{\infty}$ & $\mid \begin{array}{l}\mathscr{d} \\
\mathbb{d} \\
\alpha\end{array}$ & 总 & 옹 & $\mid \frac{1}{\Sigma}$ & 음 & Q & $\mid \frac{T}{\pi}$ & $\frac{u}{z}$ & $\mid \frac{\infty}{\infty}$ \\
\hline
\end{tabular}

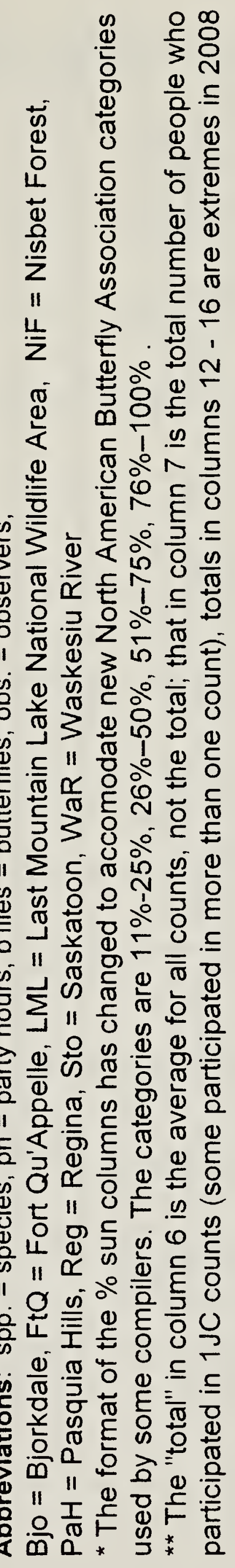


Table 2. 1JC results - Saskatchewan 2008

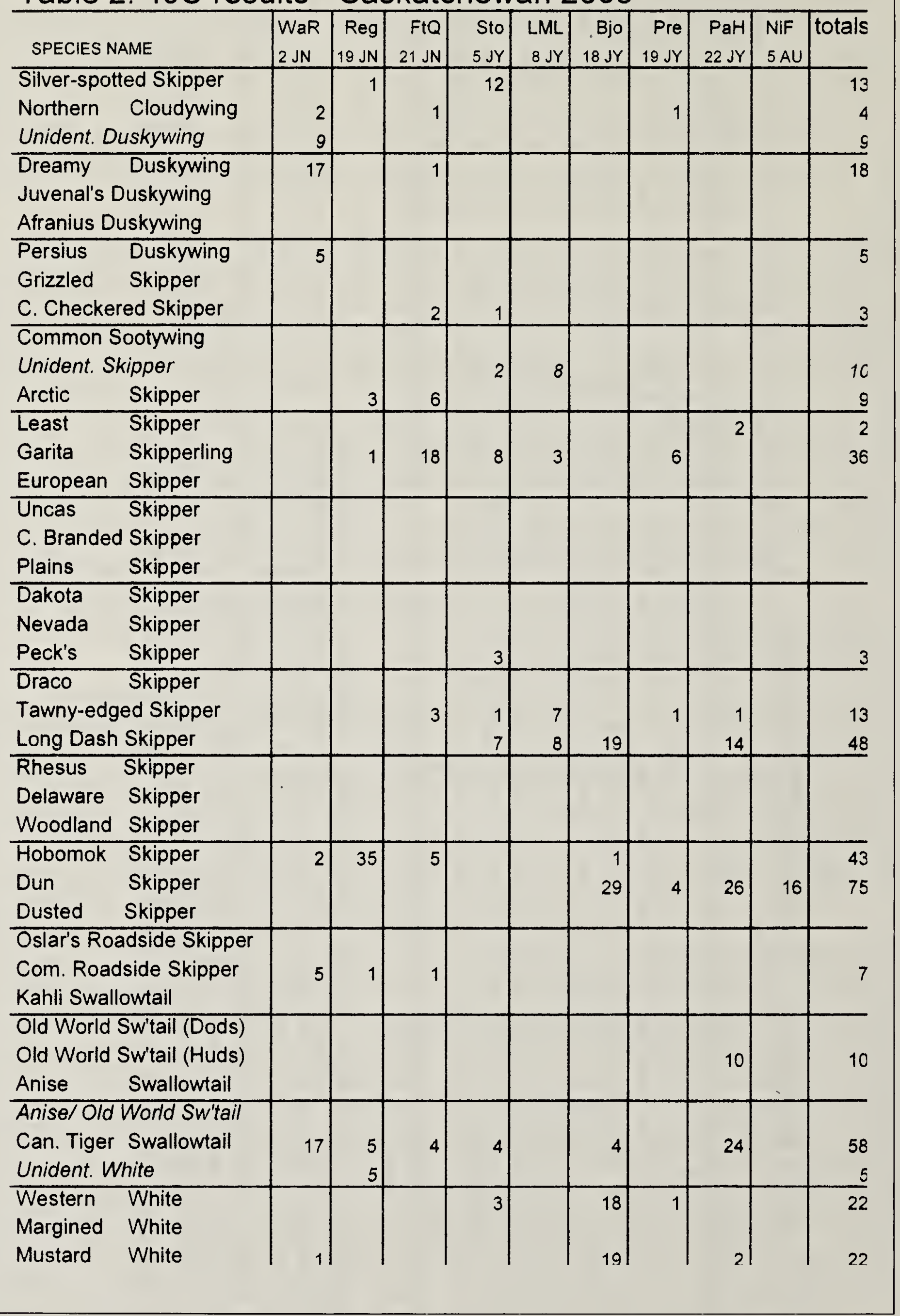




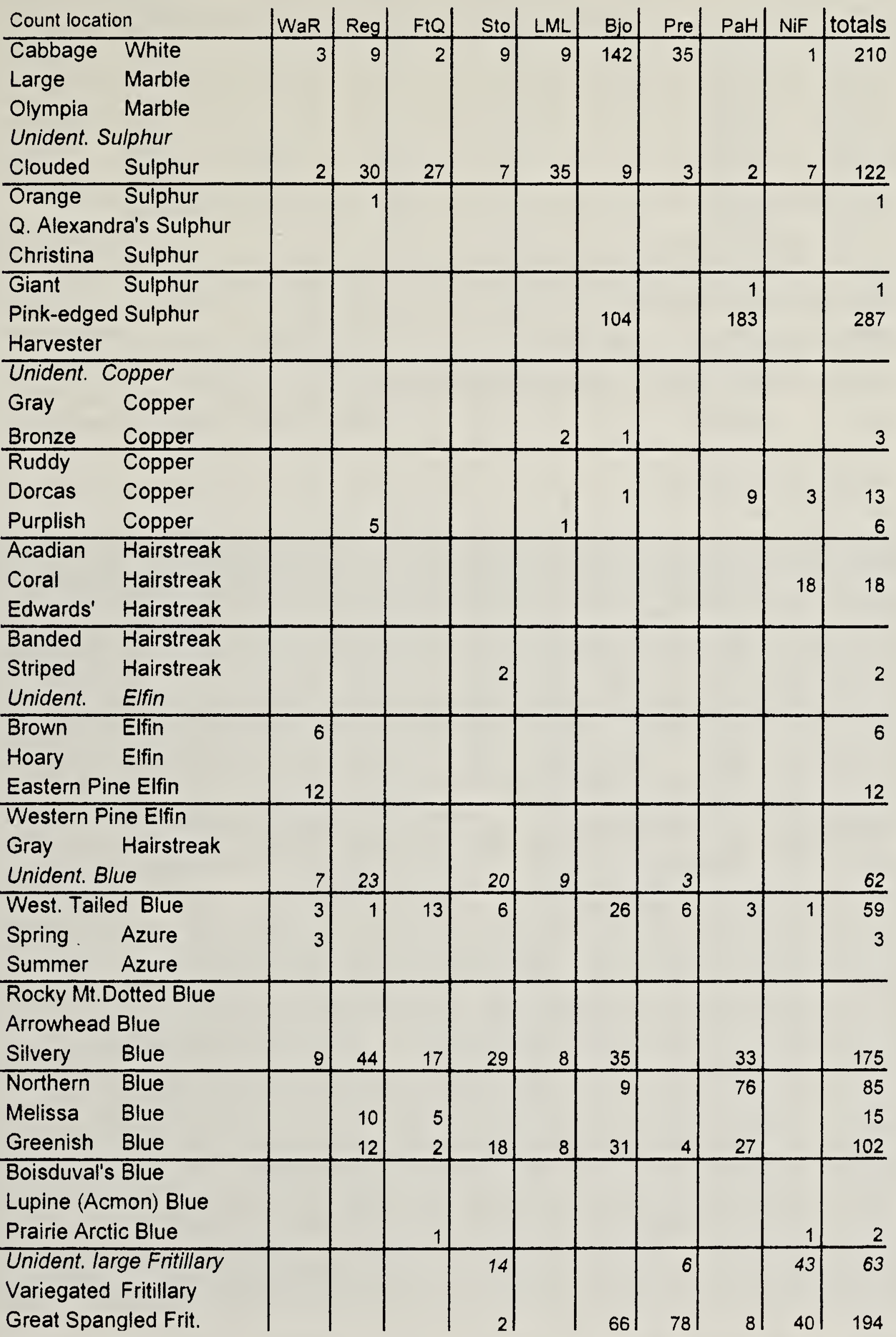




\begin{tabular}{|c|c|c|c|c|c|c|c|c|c|c|}
\hline Count location & WaR & Reg & FtQ & Sto & LML & Bjo & Pre & $\mathrm{PaH}$ & $\mathrm{NiF}$ & totals \\
\hline $\begin{array}{ll}\text { Aphrodite } & \text { Fritillary } \\
\text { Edwards' } & \text { Fritillary } \\
\text { Zerene } & \text { Fritillary }\end{array}$ & & & & & & 37 & & 13 & 16 & 66 \\
\hline $\begin{array}{ll}\text { Callippe } & \text { Fritillary } \\
\text { Atlantis } & \text { Fritillary } \\
\text { Northwestern Fritillary }\end{array}$ & & & 6 & $\begin{array}{r}3 \\
15 \\
\end{array}$ & & $\begin{array}{l}85 \\
43 \\
\end{array}$ & 2 & $\begin{array}{r}750 \\
98 \\
\end{array}$ & 13 & $\begin{array}{r}1 \\
838 \\
177 \\
\end{array}$ \\
\hline $\begin{array}{lc}\text { Mormon } & \text { Fritillary } \\
\text { Unident. small Fritillary } \\
\text { Bog } \quad \text { Fritillary }\end{array}$ & 9 & & & & & & & & 16 & 25 \\
\hline $\begin{array}{l}\text { Silver-bordered Fritillary } \\
\text { Meadow Fritillary } \\
\text { Frigga } \quad \text { Fritillary } \\
\end{array}$ & $\begin{array}{l}5 \\
3 \\
\end{array}$ & & & 1 & 1 & $\begin{array}{l}2 \\
4\end{array}$ & & $\begin{array}{r}13 \\
5\end{array}$ & $\begin{array}{l}3 \\
9\end{array}$ & $\begin{array}{r}20 \\
23 \\
3 \\
\end{array}$ \\
\hline $\begin{array}{lc}\text { Freija } & \text { Fritillary } \\
\text { Arctic } & \text { Fritillary } \\
\text { Gorgone } & \text { Checkerspot } \\
\end{array}$ & 18 & & & & 1 & & 2 & & 49 & $\begin{array}{r}18 \\
51 \\
1\end{array}$ \\
\hline $\begin{array}{l}\text { Sagebrush Checkerspot } \\
\text { Unident. Crescent } \\
\text { Pearl Crescent } \\
\end{array}$ & & $\underline{2}$ & 4 & $\begin{array}{r}37 \\
7 \\
\end{array}$ & 8 & & & & 8 & $\begin{array}{l}53 \\
13 \\
\end{array}$ \\
\hline $\begin{array}{l}\text { Northern } \quad \text { Crescent } \\
\text { Tawny } \quad \text { Crescent } \\
\text { Variable Checkerspot }\end{array}$ & 1 & 1 & 3 & $\begin{array}{r}66 \\
1\end{array}$ & 11 & $\begin{array}{r}285 \\
31\end{array}$ & $\begin{array}{r}134 \\
18\end{array}$ & $\begin{array}{r}226 \\
73\end{array}$ & $\begin{array}{r}18 \\
1\end{array}$ & $\begin{array}{l}744 \\
125\end{array}$ \\
\hline $\begin{array}{ll}\text { Unident. } & \text { Comma } \\
\text { Eastern } & \text { Comma } \\
\text { Satyr } & \text { Comma }\end{array}$ & 1 & & & 1 & & 5 & 1 & & & 13 \\
\hline $\begin{array}{ll}\text { Green } & \text { Comma } \\
\text { Hoary } & \text { Comma } \\
\text { Gray } & \text { Comma } \\
\end{array}$ & 5 & & & & & 8 & & $\begin{array}{l}2 \\
2 \\
1 \\
\end{array}$ & & $\begin{array}{l}7 \\
2 \\
9\end{array}$ \\
\hline $\begin{array}{l}\text { Unident. Nymphalids } \\
\text { Compton Tortoiseshell } \\
\text { Mourning Cloak } \\
\text { Milbert's Tortoiseshell } \\
\text { Painted Lady }\end{array}$ & $\begin{array}{l}3 \\
7\end{array}$ & 2 & & & 1 & 4 & & 3 & $\begin{array}{l}3 \\
2\end{array}$ & $\begin{array}{r}2 \\
3 \\
10 \\
9 \\
13\end{array}$ \\
\hline $\begin{array}{ll}\text { Red } & \text { Admiral } \\
\text { White } & \text { Admiral } \\
\text { Viceroy } & \\
\end{array}$ & & & 1 & 29 & 4 & $\begin{array}{r}86 \\
3 \\
\end{array}$ & 23 & $\begin{array}{r}96 \\
7 \\
\end{array}$ & 1 & $\begin{array}{r}240 \\
10 \\
\end{array}$ \\
\hline $\begin{array}{ll}\text { Northern } & \text { Pearly-Eye } \\
\text { Eyed } & \text { Brown } \\
\text { Little } & \text { Wood-Satyr }\end{array}$ & & & & & & 65 & 70 & 23 & & $\overline{158}$ \\
\hline $\begin{array}{l}\text { Common Ringlet } \\
\text { Unident. Wood-Nymph } \\
\text { Com. Wood-Nymph }\end{array}$ & & 183 & 63 & 11 & 13 & 2 & 30 & & 149 & $\begin{array}{l}272 \\
211 \\
\end{array}$ \\
\hline $\begin{array}{l}\text { Small Wood-Nymph } \\
\text { Unident. Alpine } \\
\text { Taiga Alpine }\end{array}$ & & & & & & & & & & \\
\hline
\end{tabular}




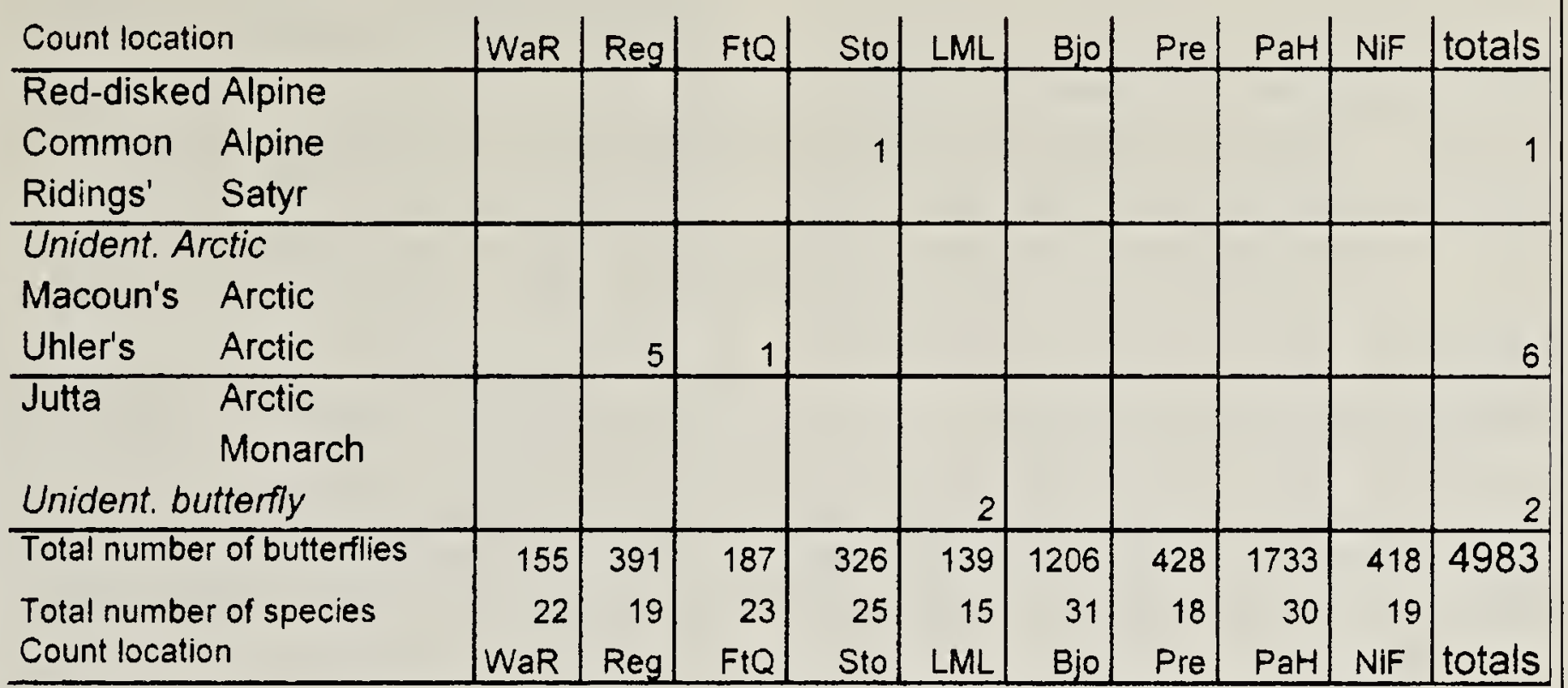

Bjo $=$ Bjorkdale, FtQ $=$ Fort Qu'Appelle, LML=Last Mt. Lake National Wildlife Area, $\mathrm{NiF}=$ Nisbet Forest, Pre $=$ Preeceville, $\mathrm{PaH}=$ Pasquia Hills, Sto $=$ Saskatoon,

Reg $=$ Regina, WaR $=$ Waskesiu River

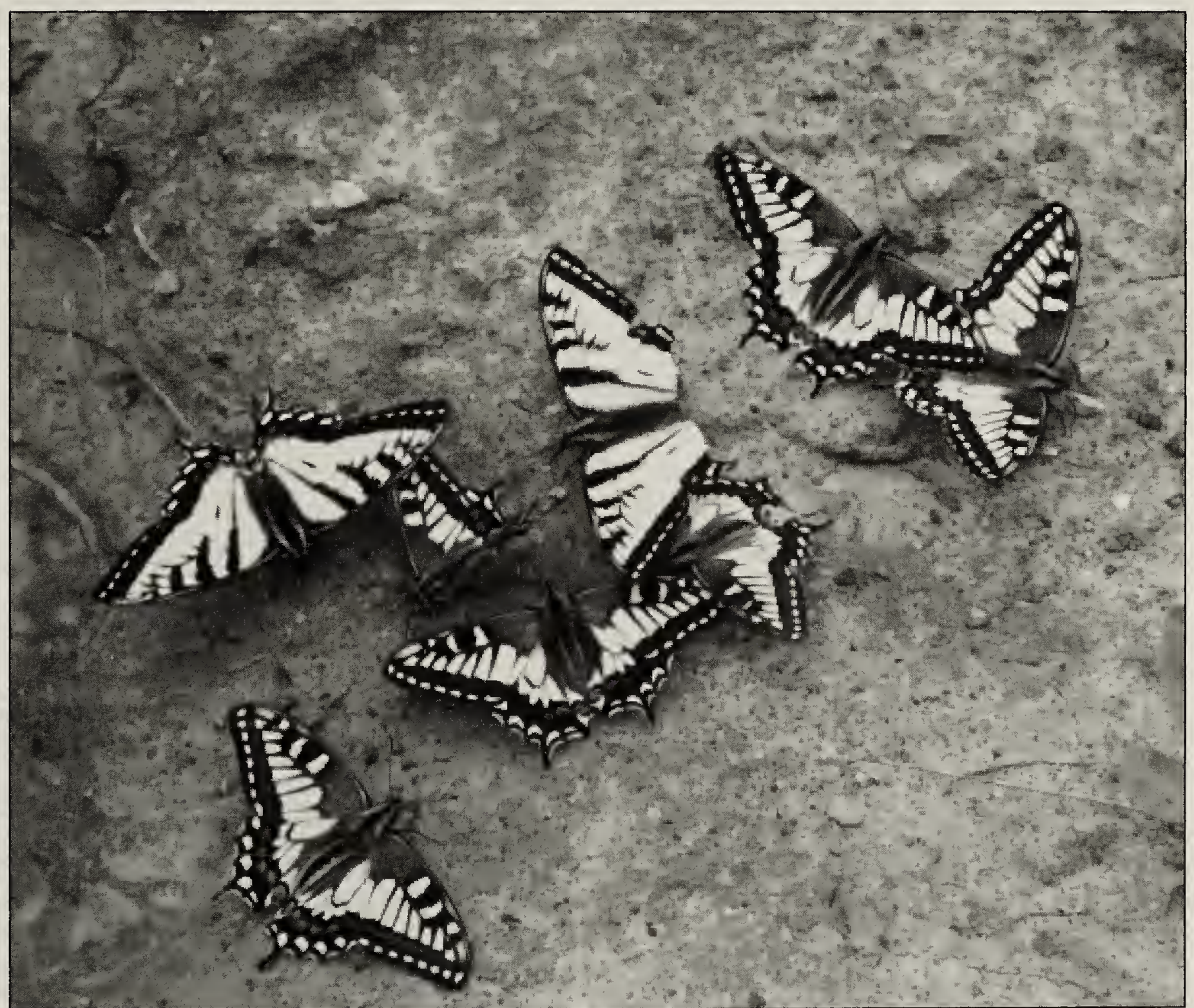

Group of Old World Swallowtails and Canadian Tiger Swallowtails, 20 June 2008, John Kozial 
The number of observers ranged from one at Bjorkdale and Pasquia Hills to 11 at Saskatoon compared to highs of 13 and 11, also at Saskatoon in 2007 and 2006 respectively. There was a total of 89 party-hours logged ranging from six ph at Nisbet Forest to 22 ph at Saskatoon. This compares with 96 party hours in 2007 and 77 in 2006.

The highest combined total from all counts of individual species in 2008 was 838 for Atlantis Fritillary, including an amazing 750 at Pasquia Hills. Other unusually high species counts at individual locations were 19 Long Dash Skippers at Bjorkdale, 29 and 26 Dun Skippers at Bjorkdale and Pasquia Hills respectively, 183 and 104 Pink-edged Sulphurs at Pasquia Hills and Bjorkdale, 18 Coral Hairstreaks at Nisbet Forest, 76 Northern Blues at Pasquia Hills, 285, 226 and 134 Northern Crescents at Bjorkdale, Pasquia Hills and Preeceville, 73, 31 and 18 Tawny Crescents at Pasquia Hills, Bjorkdale and Preeceville, 96 and 86 White Admirals at Pasquia Hills and Bjorkdale, and 70 and 65 Northern Pearly-Eyes at Preeceville and Bjorkdale.

It was a poor year for transient species. Common Checkered Skippers were present on two counts (a total of 2 individuals), Orange Sulphur on one (1) and Painted Ladies on two (12). There were no Variegated Fritillaries, Red Admirals or Monarchs.

Virtually all of the common nontransient species that might be expected considering the dates and locations of the nine counts were found in 2008 . There were no new species for the Saskatchewan 1JC. John Kozial notes "the two male specimens of Polygonia gracilis gracilis observed and collected in the Pasquia Hills $1 \mathrm{JC}$ count in 2008 represent the most southerly known collection location in the province, outside the Precambrian area. There is only one additional record (a single specimen at Candle Lake) south of the Precambrian area; that location is, however farther north than the Pasquia Hills location. There are a small number of widely scattered records for the Precambrian area of northern Saskatchewan (pers. comm. R. Hooper)." Other more unusual species noted included two Least Skippers and one Giant Sulphur at Pasquia Hills. A single Hobomok Skipper at Bjorkdale on July 18 was unusually late as was a Western-tailed Blue at Nisbet Forest on August 5. The previous late dates for Saskatchewan were July 14 and August 4 respectively.

\section{Acknowledgements}

We'd like to thank the count participants, and the count leaders and compilers Dallas Fairburn, Ron Hooper, John Kozial, Ray Poulin and Michael Williams.

1. GOLLOP, M. and A. LEIGHTON. 2005. Results of the 2005 International Butterfly Counts in Saskatchewan. Blue Jay 63:193-199

2. GOLLOP, M. and A. LEIGHTON. 2007. Results of the 2006 International Butterfly Counts in Saskatchewan. Blue Jay 65:89-95

3. GOLLOP, M and A. LEIGHTON. 2007. Results of the 2007 International Butterfly Counts in Saskatchewan. Blue Jay 65:211-217

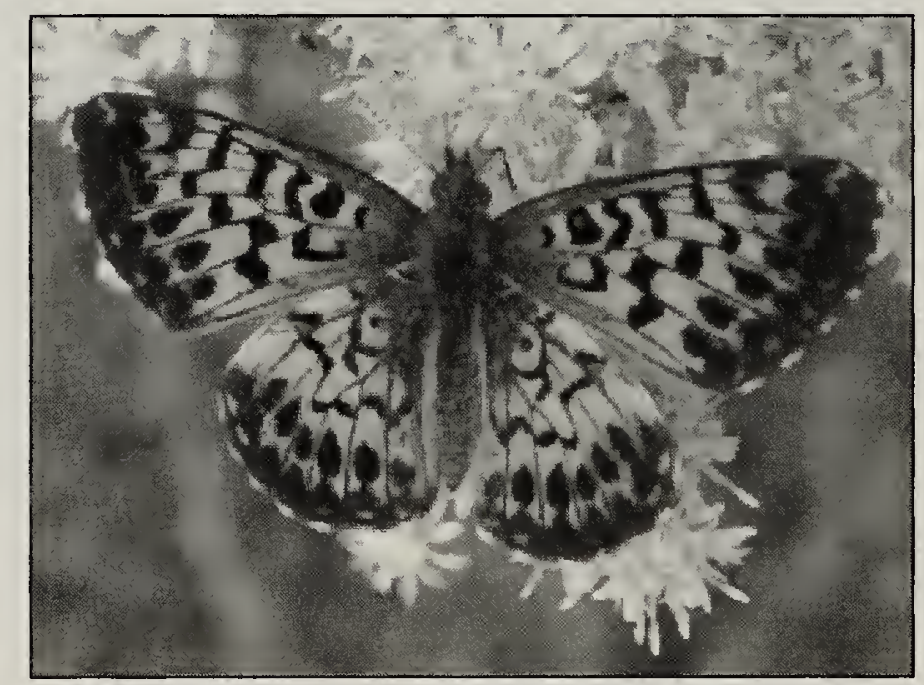

Aberrant form of Arctic Fritillary, 2 August 2008. John Kozial 